\title{
Heat and Mass Transfer Flow of Gyrotactic Microorganisms and Nanoparticles Through a Porous Medium
}

\author{
Sohail Ahmad ${ }^{1 *}$, Muhammad Ashraf ${ }^{1}$, Kashif Ali $^{2}$ \\ ${ }^{1}$ Centre for Advanced Studies in Pure and Applied Mathematics, Bahauddin Zakariya University, Multan 60800, Pakistan \\ ${ }^{2}$ Department of Basic Sciences and Humanities, Muhammad Nawaz Sharif University of Engineering and Technology, Multan \\ 60000, Pakistan
}

Corresponding Author Email: muhammadashraf@bzu.edu.pk

https://doi.org/10.18280/ijht.380215

Received: 20 March 2020

Accepted: 2 June 2020

\section{Keywords:}

nanofluids, gyrotactic microbes, heat generation, chemical reaction, porous media

\begin{abstract}
The insertion of microorganisms (microbes) in commonly used nanofluids is helpful to enhance the thermal efficiency in many systems such as bio-microsystems like chipshaped microdevices, microbial fuel cells, enzyme biosensor and microfluidics devices like micro-volumes and bacteria powered micro-mixers. The utilization of porous media is another way to enhance the thermal efficiency. Our concern in this article is to numerically investigate the heat and mass transfer flow of gyrotactic microorganisms and nanofluids with allowance for chemical reaction and heat generation through a porous medium past a nonlinear stretching sheet. The fully coupled nonlinear system of equations is solved by employing the SOR (Successive over Relaxation) method. The repercussions of preeminent parameters on the flow, heat and mass transfer as well as microorganisms diffusion rate are deliberated and shown through graphs and tables. The culminations evidently disclose that the bioconvection Peclet number and the motile microbes parameter enhance the density of the motile microorganisms. It is also found that porous medium has negligible effect on motile microbes' concentration while it significantly enhances the shear stress.
\end{abstract}

\section{INTRODUCTION}

In modern times, research community has made tremendous efforts to enrich the theoretical models, experimental work and numerical simulations of nanofluids. Kumar and Varma [1] interrogated the hydro-magnetic flow of nanofluid through a porous medium past an extending sheet with variable wall thickness in the existence of Brownian motion. It was found that the heat diffusion increases due to strong magnetic field and it causes the devaluation in heat transfer rate. Stagnation point nanofluid mixed convective electro magneto hydrodynamics (EMHD) flow through a permeable medium with the impact of joule heating over a stretching sheet was premeditated by Lakshmi et al. [2]. The governing PDEs along with BCs were transformed into ODEs and were settled numerically by exploiting the Keller Box method. Both the electric and magnetic parameter increased the temperature distribution at some specific points in the flow region. An investigation is accomplished by Mabood et al. [3] on the flow of water based nanofluids $\left(\mathrm{Al}_{2} \mathrm{O}_{3}\right.$ and $\left.\mathrm{Cu}\right)$ with mass and heat transfer under the impacts of radiation, chemical reaction and viscous dissipation. Umadevi and Nithyadevi [4] exploited the finite volume method along with power-law scheme to scrutinize the MHD convection of water-based nanofluids on various thermal boundaries in an enclosure with the influence of heat generation. Fluids committed in this analysis were $A g$-water, pure water and $\mathrm{TiO}_{2}$. It was observed that the thermal conductivity of $T i$ nanoparticle is less than that of $A g$ particle. An analysis of MHD axisymmetric micropolar nanofluid flow through porous parallel disks was presented by Abbas et al. [5]. Their results spotted that the radial velocity curves rise up near the disks and diminish at the central plane. The flow of nanofluids over stretching/shrinking surfaces are much eminent in the industrial fields such as extrusion process of aerodynamics, manufacturing of glass fibers, plastic and rubber sheets manufacturing, metallic plates cooling process, cooling or drying process of papers, cooling process of nuclear reactors and MHD power generators.

Recently, the intention of scholars is deflected towards flow of gyrotactic microorganisms within nanofluids. Kuznetsov [6] commenced the abstract work of bioconvection nanofluid incorporating gyrotactic microorganisms and declared that the self-propelled motile microbes strengthen mixing and avert nanoparticles accumulation in nanofluids. Being a recently discovered phenomenon, occurrence of bioconvection is the utmost common event involving the microscopic movement of motile microbes that move up (swim upward) like Oxytactic bacteria, Chlamydomonas nivalis and so on. Bioconvection takes place because the microbes are slightly denser than water and can float causing a very dense volatile state due to the microorganisms array on the upper surface of the water. The premises of this apparent fact may not be the same for all species due to the involvement of some factors such as thermal radiation, chemical reaction, gravity, heat generation and viscous dissipation that specify the swimming in a certain direction. Microorganism's particles are widely used to prepare the commercial and industrial items like bio fuel made from waste, fertilizers, ethanol etc. They are, 
additionally, utilized in water treatment plants. Promising sustainable power sources (biodiesel and hydrogen gas) can be generated by the microorganisms. These are also valuable in providing nutrients to plants, delivering oxygen, maintaining human health and decomposing organic material. But some of these organisms may be pathogenic and cause serious infections in humans and plants. Recently, a novel type of microorganism (which is a virus named coronavirus) has been traced out [7], and is given the provisional name 2019-nCOV by the WHO (World Health Organization). This virus has been declared as Pandemic and the organization (WHO) named the disease it causes COVID-19. A coronavirus is a type of typical virus that reasons an infection in sinuses, nose or upper throat.

Effects of gyrotactic microbes in nanofluid flows have been intensively studied. Ramzan et al. [8] analyzed the mass and heat transfer in both nanofluid and gyrotactic microorganisms. This study included viscous dissipation with convective boundary conditions, slip effects and non-linear thermal radiation. It was observed that microorganisms motile density declines with climbing values of the Lewis and Peclet numbers. In an investigation on nanofluid flow comprising gyrotactic microorganisms under the impact of external magnetic field by Chakraborty et al. [9], it was found that the heat transport rate, momentum boundary layer thickness and the flux rate of motile microorganisms across the fluid medium diminish with an increment in the magnetic field parameter while the nanoparticle concentration enhances. An effort was made by Tausif et al. [10] to explore the nanofluid flow containing nanoparticles and microorganisms with multiple slip effects. In this study, it was suggested that by escalating the values of various slip parameters, fluid properties like microorganism flux rate and heat/mass transfer rate can be reduced or improvised to achieve the required quality of the final product in polymer industry. The steady two dimensional nanofluid flow consisting of both gyrotactic microbes and nanoparticles was observed by Iqbal et al. [11]. This study comprised stagnation point flow of circuitously striking nanofluid. Atif et al. [12] avowed that motile microorganism density profile uplifts with the buoyancy ratio parameter and depreciates with micropolar parameters. They incorporated the effects of thermal radiation and Joule heating in MHD bioconvective stratified micropolar fluid flow comprising gyrotactic microorganisms. Acharya et al. [13] examined that the motile microbes density significantly elevates with the Lewis and bioconvective Peclet numbers. They adopted the Keller box method for numerical simulation and pointed out that the amplifying values of Péclet number tend to decrease the motile microorganisms concentration. Various interesting characteristics like nonlinear radiation, gyrotactic microorganisms and Arrhenius energy activation in the flow of electrically conducted Maxwell nanofluid were explored by Waqas et al. [14].

One of the objectives of this work is to explore whether bioconvection (in a nanofluid flow comprising gyrotactic microbes) can take place in a permeable media. To the best of our knowledge, this work presents first effort to investigate the mass and heat transportation flow comprising gyrotactic microbes and nanofluids with allowance for heat generation and chemical reaction through a porous media over a nonlinear stretching sheet. Besides, our proposed method (SOR) is used for the first time to solve the dynamical problem of nanofluids involving gyrotactic microbes. The effects of physical parameters on the flow velocity, concentration, temperature and mobile microbes' density distribution are examined and visualized graphically.

\section{DESCRIPTION OF PHYSICAL MODEL}

Let us assume two-dimensional and steady nanofluid flow through porous medium enclosing both, gyrotactic microbes and nanoparticles, by taking the impacts of heat generation and chemical reaction. $N_{w}, C_{w}$ and $T_{w}$ denote the concentration of the microorganisms, concentration and temperature of fluid at sheet surface (see Figure 1). It is supposed that the velocity of swimming microbes as well as swimming path way do not possess any change in the presence of nanoparticles. This assumption is valid only if concentration of nanoparticles is below than $1 \%$. Thereby, bioconvection stability can be acquired by mixing solid nano particles along with microbes in the liquid (base fluid). Momentum, species and thermal boundary layers across the flow region are shown in the flow sketch Figure 1.

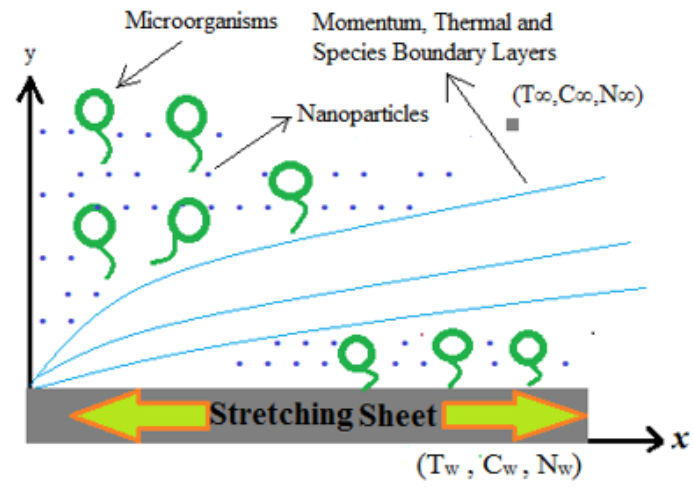

Figure 1. Geometry of the problem

The differential equations governing the flow model (in case of porous media, heat generation and chemical reaction) have the form [15]:

$$
\begin{gathered}
\frac{\partial u}{\partial x}+\frac{\partial v}{\partial y}=0 \\
u \frac{\partial u}{\partial x}+v \frac{\partial u}{\partial y}=v \frac{\partial^{2} u}{\partial y^{2}}+u_{e} \frac{\partial u_{e}}{\partial x}+\frac{\mu}{\rho k^{*}}\left(u_{e}-u\right) \\
u \frac{\partial T}{\partial x}+v \frac{\partial T}{\partial y}=\frac{\tau D_{T}}{T_{\infty}}\left(\frac{\partial T}{\partial y}\right)^{2}+\tilde{\alpha} \frac{\partial^{2} T}{\partial y^{2}}+\frac{Q_{0}}{\rho c_{p}}\left(T-T_{\infty}\right) \\
+\tau D_{B} \frac{\partial C}{\partial y} \frac{\partial T}{\partial y} \\
u \frac{\partial C}{\partial x}+v \frac{\partial C}{\partial y}=\frac{D_{T}}{T_{\infty}} \frac{\partial^{2} T}{\partial y^{2}}+D_{B} \frac{\partial^{2} C}{\partial y^{2}}-K_{c}\left(C-C_{\infty}\right) \\
u \frac{\partial N}{\partial x}+v \frac{\partial N}{\partial y}+\frac{1}{C_{w}-C_{\infty}} \frac{\partial}{\partial y}\left[N\left(\frac{\partial C}{\partial y}\right)\right] b W_{c} \\
=D_{n} \frac{\partial^{2} N}{\partial y^{2}}
\end{gathered}
$$

where, $x$ and $y$ be the coordinates of the axes along and normal to the sheet withuand $\mathrm{v}$ being the respective velocity 
components in the $x$ and $y$ directions. Moreover $k^{*}, \rho, T_{\infty}, v$, $Q_{0}, N, T, C, b W_{c}, \tau, D_{T}, c_{p}, C_{\infty}, D_{B}, \tilde{\alpha}, K_{c} \& D_{n}$ represent the Darcy permeability, density, ambient temperature, kinematic viscosity, heat generation coefficient, microorganism concentration, temperature, concentration of fluid, cell swimming speed, nanofluid heat capacity ratio, thermophoretic coefficient, specific heat constant, ambient concentration, Brownian diffusion coefficient, thermal conductivity, rate constant of chemical reaction and diffusivity of microorganisms respectively. The following analogous boundary conditions (BCs) at sheet surface $(\mathrm{y}=0)$ and far from sheet $(\mathrm{y} \rightarrow \infty)$ are imposed as:

$$
\begin{gathered}
\begin{array}{r}
y=0: u=c x^{m}, N=N_{w}, C=C_{w}, T=T_{w}, v \\
=v_{w}(x)
\end{array} \\
y \rightarrow \infty: u=a x^{m}, N=N_{\infty}, C=C_{\infty}, T=T_{\infty}
\end{gathered}
$$

where, $v_{w}$ is mass flux which corresponds to suction $\left(v_{w}>0\right)$ and injection $\left(v_{w}<0\right)$. Where as $u_{e}(x)=a x^{m}$ is to be taken as ambient velocity and that of $u_{w}(x)=c x^{m}$ is stretching velocity, where $m$ is positive constant other than one (due to nonlinear shrinking/stretching sheet). In addition, $N_{\infty}, C_{\infty}$ and $T_{\infty}$ denote the concentration of the microorganisms, concentration and temperature of fluid far away from sheet surface. Following similarity transformations are introduced along with stream function to transmute the governing PDEs (1)-(5) into ODEs.

$$
\begin{aligned}
\psi=\sqrt{a x^{m+1}} v f(\xi), \xi=\sqrt{\frac{a}{v} x^{m-1}} y, G(\xi) \\
=\frac{N-N_{\infty}}{N_{w}-N_{\infty}} \phi(\xi)=\frac{C-C_{\infty}}{C_{w}-C_{\infty}}, \theta(\xi) \\
=\frac{T-T_{\infty}}{T_{w}-T_{\infty}}
\end{aligned}
$$

Entreating these variables in Eqns. (2)-(5), we acquire the following set of equations:

$$
\begin{gathered}
f^{\prime \prime \prime}+P_{0}\left(1-f^{\prime}\right)=m\left(f^{\prime 2}-1\right)-\frac{m+1}{2} f f^{\prime \prime} \\
\frac{1}{\operatorname{Pr}} \theta^{\prime \prime}+N_{b} \theta^{\prime} \phi^{\prime}+\frac{m+1}{2} f \theta^{\prime}+H \theta+N_{t} \theta^{\prime 2}=0 \\
\phi^{\prime \prime}+\frac{m+1}{2} f \theta^{\prime}+\frac{N_{t}}{N_{b}} \theta^{\prime \prime}=L e R_{c} \phi \\
G^{\prime \prime}+\frac{m+1}{2} S c f G^{\prime}=\operatorname{Pe}\left[\phi^{\prime} G^{\prime}+(G+\Omega) \phi^{\prime \prime}\right]
\end{gathered}
$$

The interrelating BCs (6) and (7) become now:

$$
\begin{gathered}
\xi=0: f=S=1, f^{\prime}=\alpha, \phi=1, \theta=1 \\
\xi \rightarrow \infty: f^{\prime} \rightarrow 1, \phi \rightarrow 0, \theta \rightarrow 1, G \rightarrow 0,
\end{gathered}
$$

where, $\alpha$ is the shrinking (if $\alpha<0$ ) or stretching (if $\alpha>0$ ) of sheet parameter, $S$ is suction/injection and the other parameters involved in the system of Eqns. (9)-(12) are:

$P_{0}=\frac{v x}{k^{*} u_{e}}$ is porosity parameter,

$N_{b}=\frac{\tau D_{B}\left(C_{w}-C_{\infty}\right)}{v}$ is Brownian motion parameter,
$\Omega=\frac{N_{\infty}}{N_{W}-N_{\infty}}$ is motile microbes parameter,

$S c=\frac{v}{D_{n}}$ is Schmidt number, $\operatorname{Pr}=\frac{v}{\alpha}$ is Prandtl number,

$N_{t}=\frac{\tau D_{T}\left(T_{w}-T_{\infty}\right)}{v T_{\infty}}$ is thermophoresis parameter,

$L e=\frac{v}{D_{B}}$ is Lewis number,

$P e=\frac{b W_{c}}{D_{n}}$ is the bioconvection Peclet number,

$R_{c}=\frac{2 K_{c} x}{u_{e}}$ is chemical reaction parameter,

$H=\frac{Q_{0} x}{\rho c_{p} u_{e}}$ is heat generation parameter.

The local density number of motile microbes is

$$
\operatorname{Re}_{\mathrm{x}}^{\frac{-1}{2}} \mathrm{~N}_{\mathrm{n}}(\mathrm{x})=-\mathrm{G}^{\prime}(0)
$$

\section{NUMERICAL ANALYSIS}

We construct numerical algorithm of our problem by using finite difference based technique. As suggested by Ashraf and Bashir [16], we recede the order of Eq. (9) by modifying:

$$
s=f^{\prime}=\frac{d f}{d \xi}
$$

So that we have to solve the nonlinear Eqns. (9)-(12) in the following form:

$$
\begin{gathered}
s^{\prime \prime}+P_{0}(1-s)=m\left(s^{2}-1\right)-\frac{m+1}{2} f s^{\prime} \\
\frac{1}{\operatorname{Pr}} \theta^{\prime \prime}+N_{b} \theta^{\prime} \phi^{\prime}+\frac{m+1}{2} f \theta^{\prime}+H \theta+N_{t} \theta^{\prime 2}=0 \\
\phi^{\prime \prime}+\frac{m+1}{2} f \theta^{\prime}+\frac{N_{t}}{N_{b}} \theta^{\prime \prime}=L_{e} R_{c} \phi \\
G^{\prime \prime}+\frac{m+1}{2} S c f G^{\prime}=\operatorname{Pe}\left[\phi^{\prime} G^{\prime}+(G+\Omega) \phi^{\prime \prime}\right]
\end{gathered}
$$

With BCs:

$$
\begin{gathered}
f(0)=1, s(0)=\alpha, G(0)=\theta(0)=\phi(0)=1 \\
s(\infty)=1, G(\infty)=\theta(\infty)=\phi(\infty)=0
\end{gathered}
$$

After using finite differences, Eqns. (16)-(19) take the form:

$$
\begin{aligned}
s_{i} & =\frac{1}{A_{1}}\left(B_{1} s_{i+1}+C_{1} s_{i-1}+D_{1}\right) \\
\theta_{i} & =\frac{1}{A_{2}}\left(B_{2} \theta_{i+1}+C_{2} \theta_{i-1}+D_{2}\right) \\
\phi_{i} & =\frac{1}{A_{3}}\left(B_{3} \phi_{i+1}+C_{3} \phi_{i-1}+D_{3}\right) \\
G_{i} & =\frac{1}{A_{4}}\left(B_{4} G_{i+1}+C_{4} G_{i-1}+D_{4}\right)
\end{aligned}
$$

The values of constants $\left(A_{l}, B_{l}, C_{l}\right.$ etc. $)$ are given in appendix. 
The following iterative procedure is applied to acquire the numerical solution of the problem [17].

(1). An initial guess for $\hat{S}^{(0)}, \hat{G}^{(0)}, \hat{\theta}^{(0)}$ and $\hat{\phi}^{(0)}$ is chosen to satisfy the boundary conditions (20) and (21).

(2). The approximation $\hat{f}^{(1)}$ is found by solving the linear system (15) with the help of Simpson's rule, which is then utilized to determine $\hat{S}^{(1)}$ in Eq. (22).

(3). The derivatives in Eqns. (16)-(19) are replaced by finite differences, and the resultant algebraic system (22)-(25) is solved by SOR to find the approximations $\hat{S}^{(1)}, \hat{G}^{(1)}, \hat{\theta}^{(1)}$ and $\hat{\phi}^{(1)}$.

After the functions $\hat{S}^{(1)}, \hat{G}^{(1)}, \hat{\theta}^{(1)}$ and $\hat{\phi}^{(1)}$ are determined, the procedure is repeated until the sequences $\left\{\hat{S}^{(k)}\right\},\left\{\hat{G}^{(k)}\right\},\left\{\hat{\theta}^{k}\right\}$ and $\left\{\hat{\phi}^{(k)}\right\}$ converge to $\hat{s}, \hat{g}, \hat{\theta}$ and $\hat{\phi}$ respectively.

\section{RESULTS AND DISCUSSIONS}

In this section, we interpret the results by means of physical interpretations. The nonlinear governing Eqns. (16)(19) have been solved numerically for diverse values of the governing parameters subject to the BCs (20). A finitedifference based persuasive technique known as the SOR method, as described in the book by Hildebrand [18], is used to obtain the numerical solutions. The step size of $\xi$ as well as the edge of the boundary layer are balanced in such a way that the velocity $F^{\prime}(\xi)$, temperature $\theta(\xi)$, concentration $\phi(\xi)$ and motile microbes density distribution $G(\xi)$ show asymptotic appearance for various scopes of physical parameters. Numerical results are related with the earlier ones (see Table 1) and, the assessment is observed to be in a good agreement which confirms the accuracy of our computational procedure. Table 1 also indicates that values of the physical quantity such as motile microbes density number elevate with an increment in suction/injection parameter $S$.

Table 1. Comparison with $N n_{x}$ for various $S$

\begin{tabular}{ccc}
\hline $\boldsymbol{S}$ & Zaimi et al. [19] & Present \\
\hline 2.5 & 5.48217 & 5.4748 \\
3.0 & 6.49841 & 6.4862 \\
3.5 & 7.54285 & 7.5237 \\
4.0 & 8.60180 & 8.5736 \\
\hline
\end{tabular}

As suggested by Ahmad et al. [20], a best way to analyze the flow characteristics of a dynamical problem is to fix the values to non-dimensional parameters rather than assigning the particular fluid properties and the domain dimensions. So, we assign fixed values to the parameters such as $P_{0}=20$, $H=0.6, S C=1.4, N_{t}=0.25, P e=0.5, L e=2, P r=4.2, \Omega=0.22, S=1$, $m=2.5, N_{b}=0.2, R_{c}=1$ and $\alpha=0.6$ otherwise specified. From Table 2, it is obvious that the impact of positive constant $m$ is to escalate the skin friction as well as local motile microbes density.

If porosity is small then dilute suspension may not generate required concentration for which bioconvection occurs. It is observed in the present case that if we go on increasing the values of porosity parameter then we acquire better solution for bioconvection parameters. As size of pores is large $\left(P_{0}>0\right)$ so, porous media does not absorb microbes and thus no significant change is seen in motile microbes concentration $N n_{x}$ (please see Table 3).
Table 2. $C_{f x}$ and $N n_{x}$ for various $m$

\begin{tabular}{ccc}
\hline $\boldsymbol{m}$ & $\boldsymbol{C}_{\boldsymbol{f} \boldsymbol{x}}$ & $\boldsymbol{N} \boldsymbol{n}_{\boldsymbol{x}}$ \\
\hline 3 & 2.4735 & 3.2163 \\
4 & 2.6684 & 3.5202 \\
5 & 2.8637 & 3.8420 \\
6 & 3.0594 & 4.1807 \\
\hline
\end{tabular}

Table 3. $C_{f x}$ and $N n_{x}$ for various $P_{0}$

\begin{tabular}{ccc}
\hline $\boldsymbol{P}_{\boldsymbol{0}}$ & $\boldsymbol{C}_{\boldsymbol{f} \boldsymbol{x}}$ & $\boldsymbol{N} \boldsymbol{n}_{\boldsymbol{x}}$ \\
\hline 0 & 1.3373 & 3.0292 \\
1 & 1.4131 & 3.0333 \\
10 & 1.9452 & 3.0570 \\
100 & 4.4252 & 3.1066 \\
\hline
\end{tabular}

The results designate that local motile microbes density $\left(N n_{x}\right)$ which is caused by the bioconvection phenomenon is stable only when porosity is large.

Bioconvection phenomenon takes the fluid towards sheet and, as a result, motile microbes density enhances on surface. An enhancement in the motile microbes' density number $\left(N n_{x}\right)$ is observed on sheet surface with an increase in $\Omega$ as predicted in Table 4. If concentration of nanoparticles at the surface of sheet is high as compared to the concentration of fluid then transfer of mass occurs from sheet to the fluid. So, an enhancement in $\left(N n_{x}\right)$ may be attributed to the reason that concentration is low on the sheet. It can be noticed that the porous medium enhances the shear stress and that of bioconvection parameter $\Omega$ enhance the density of the motile microbes respectively. These results lead to suggest that the shear stresses, mass and heat transportation rates as well as mobile microbes diffusion rate may be adjusted by appropriately selecting parametric values and suction/injection velocity at the surface of sheet to attain the required consequences in factual applications of the present problem.

Table 4. $C_{f x}$ and $N n_{x}$ for various $\Omega$

\begin{tabular}{cc}
\hline $\boldsymbol{\Omega}$ & $\boldsymbol{N n}_{\boldsymbol{x}}$ \\
\hline 2.0 & 7.2835 \\
2.5 & 7.9749 \\
3.0 & 8.6664 \\
3.5 & 9.3579 \\
\hline
\end{tabular}

It is notable that $\alpha>0$ denotes the stretching of sheet while $\alpha<0$ corresponds to the shrinking case. Figures 2 and 3 describe the demeanor of velocity and motile microbes density profiles for multiple values of $m$ and $\alpha$. Both the shrinking $(\alpha=-1)$ and stretching $(\alpha=0.5)$ of sheet along with positive constant $m$ raise the velocity $F^{\prime}(\xi)$ but depreciate microorganisms density $G(\xi)$ across the boundary layer. The nonlinearly shrinking/stretching sheet is subsidized to accelerate the flow velocity that can be observed from Figure 2 . Figure 4 reveals the impact of the porosity parameter $P_{0}$ and suction/injection parameter $S$ on streamwise velocity. An increment in $P_{0}$ and $S$ substantially uplifts the velocity $F^{\prime}(\xi)$.

Temperature $\theta$ is portrayed in Figures 5 and 6 as function of the coordinate $\xi$ for several values of $\mathrm{Pr}, H$ and $N_{t}$. The impact of $\mathrm{Pr}$ is to downturn the temperature curve, as envisioned in Figure 5. The ratio of thermal diffusivity and conductivity refers to the Prandtl number. In this way, Prandtl number with least value infers the most elevated thermal diffusivity whereas higher Prandtl number prompts 
larger diffusivity. This reality brings about lower temperature as well as boundary layer thickness. Whereas an opposite trend in temperature profile is noted in case of heat generation and thermophoretic parameters, that is, both these parameters increase the temperature (see Figure 6). An increment in $H$-values causes more heat transport to the fluid and substantially elevates the temperature as well as thermal boundary layer thickness.

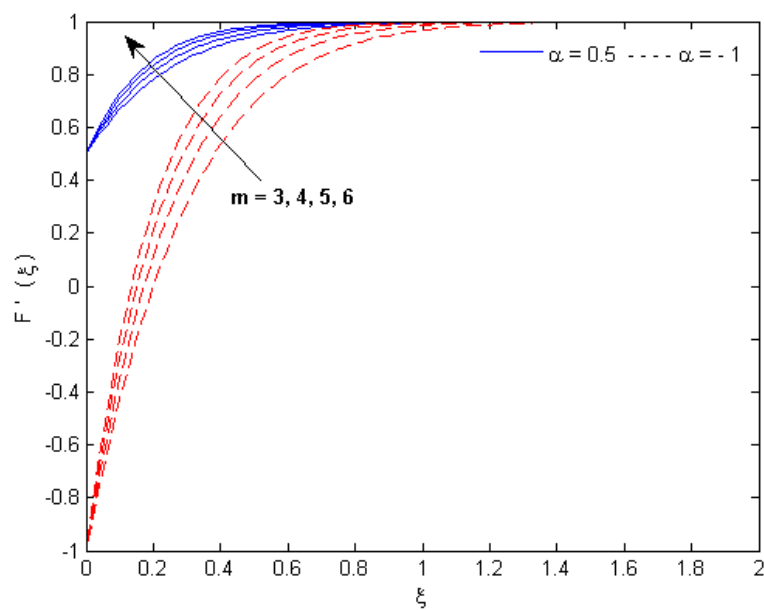

Figure 2. Velocity profile for various $m$ and $\alpha$

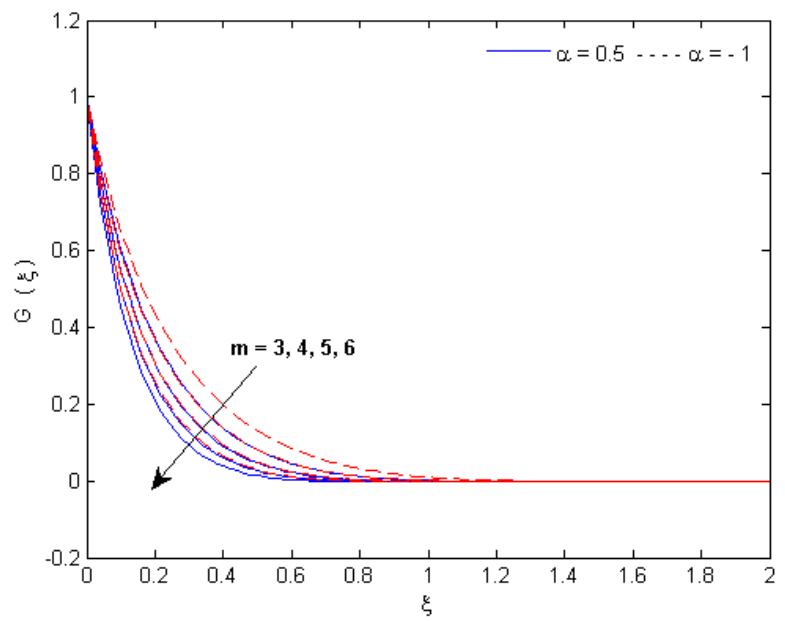

Figure 3. Motile microorganisms density profile for various $m$ and $\alpha$

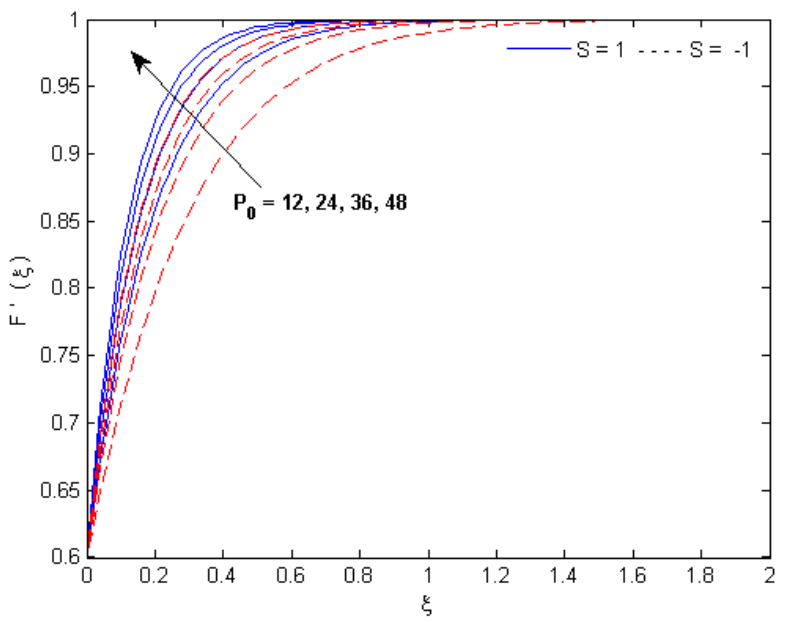

Figure 4. Velocity profile for various $P_{o}$ and $S$

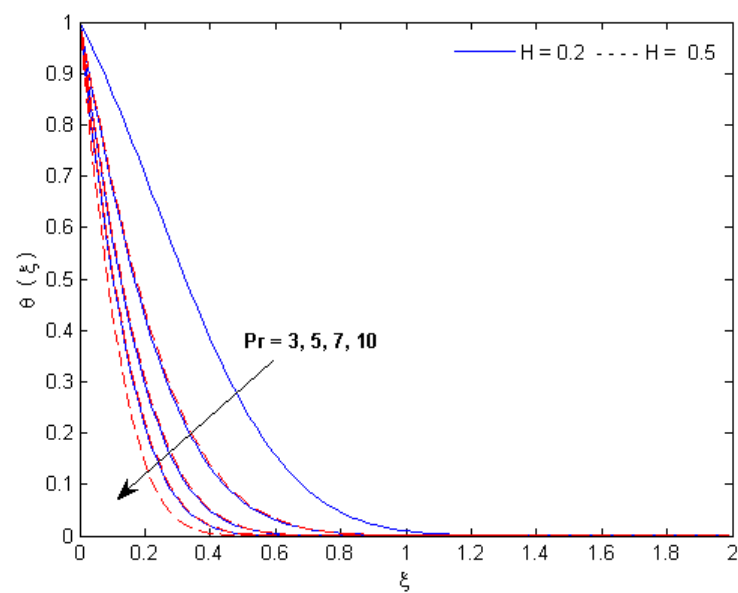

Figure 5. Temperature profile for various $\operatorname{Pr}$ and $H$

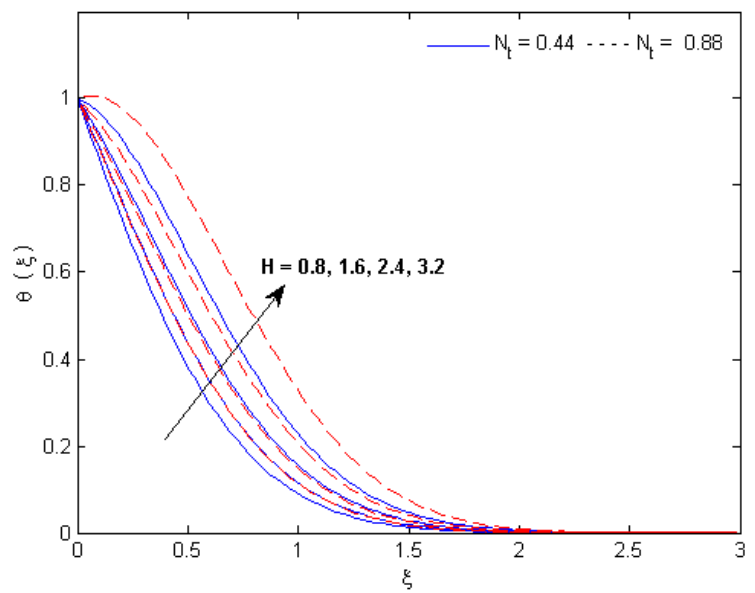

Figure 6. Temperature profile for various $H$ and $N_{t}$

Figure 7 is drawn against the concentration $\phi(\xi)$ for various $R_{c}$ and $N_{b}$. The results of Figure 7 depict that an enhancement in the chemical reaction and Brownian motion parameter leads to a decrease in the concentration profile $\phi(\xi)$.

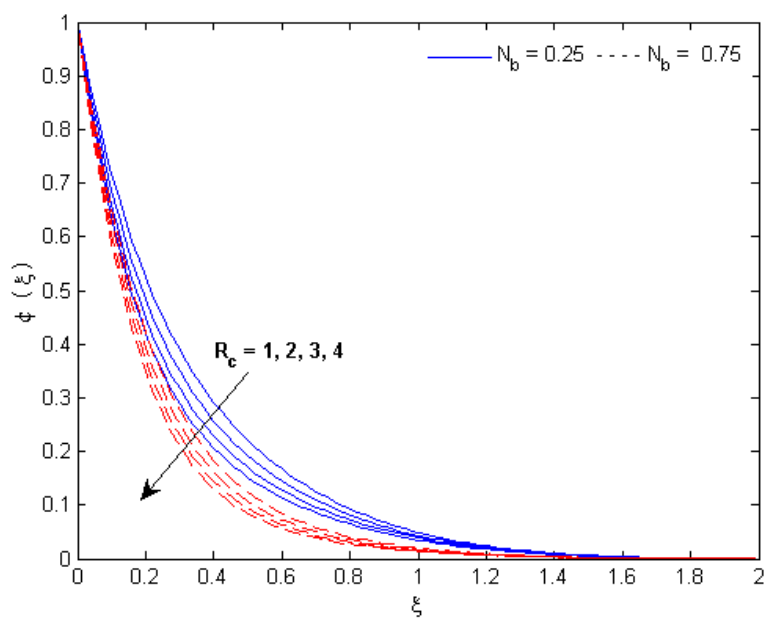

Figure 7. Concentration profile for various $R_{c}$ and $N_{b}$

Contrary to the motile microbes, the nanoparticles do not possess self-propelled movement. The motion of motile microbes is driven by bioconvection while movement of 
nanoparticles is due to the thermophoresis and Brownian motion. Thereby, movement of the nanoparticles is independent of the microbes. It may be better to study phytoplankton (most of which are single-celled plants) and Chlamydomonas (which is single-celled green algae) as model gyrotactic microbes. Both are microscopic, photosynthetic and green coloured organisms found in aquatic environment, and are helpful to study the gyrotactic microbes' movement in fluid flows. Actively mobile phytoplankton and Chlamydomonas move upward along the gravitational force. Self-propelled movement of these microbes in a fluid flow is shown in Figure 8 (microscopic view).

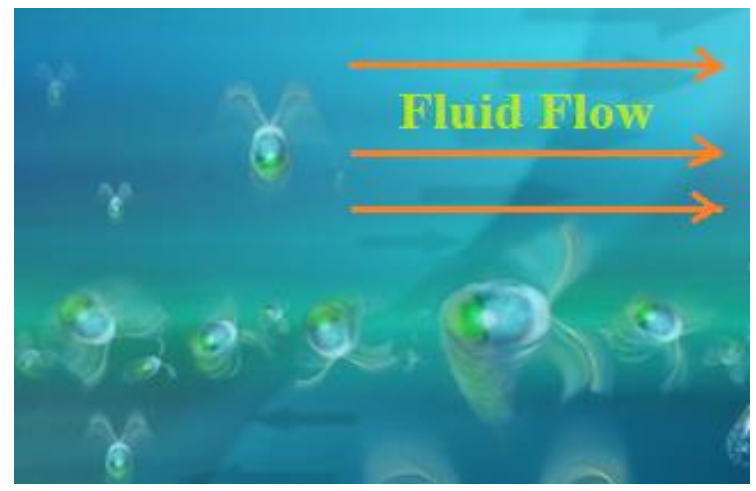

(a) Chlamydomonas

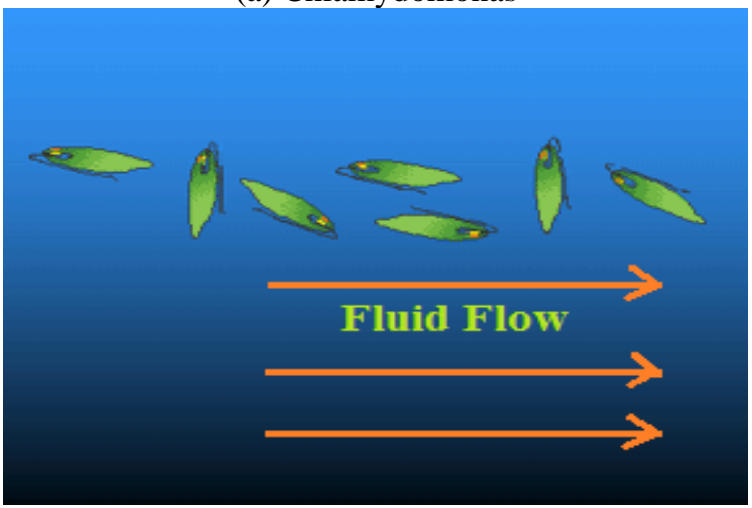

(b) Phytoplankton

Figure 8. Motion of gyrotactic microorganisms

Mostly microorganisms do not have the ability to survive at high temperatures or radiation. But some microbes can exist at extremely high temperatures, near hydrothermal vents, and at extremely low temperatures inside polar sea ice. For example, growth and total lipid production of green microalgae, Chlamydomonas reinhardtii (which are microorganisms) can be increased at high temperature and then they can be potentially utilized as novel raw materials for the production of biodiesel. Microorganisms such as thermophiles (e.g. Bacteria and Archaea) are the organisms that thrive at relatively high temperatures between $41{ }^{\circ} \mathrm{C}$ and $122{ }^{\circ} \mathrm{C}$. Bacteria present in milk and water are resistant to high temperature. Some most resistant to radiation microorganisms are escheririchia coli, streptococcus thermophiles, bifidobacteria, streptococcus aureus and lactobacilli.

Bioconvection take place because of the unstable density stratification, that is, it is brought about by the up swimming of motile microbes like chlaymadomonas which arrange (on the upper surface of the fluid) a concentrated layer. In the concerned problem, motion of the motile microbes is due to gravitational torques and viscous drag in the flow (gyrotaxis). The behavior of the motile microbes' distribution for distinct values of the bioconvection Schmidt and Lewis number has been examined in Figure 9. The microorganisms concentration profiles depress with escalating values of $S c$ and $L e$. It is noticed that the profiles $\phi(\xi)$ as well as $G(\xi)$ are substantially diminished by expanding the estimations of $L e$. This may occur because of the way that by expanding the estimations of $L e$ there is decline in the nanofluid mass diffusivity which lessens the concentration of nanoparticles. Besides, as the bioconvection $L e$ enhances, the rate of viscous diffusion builds which lessens the fluid speed at the sheet surface, and likewise diminishes the density distribution of the motile microorganisms.

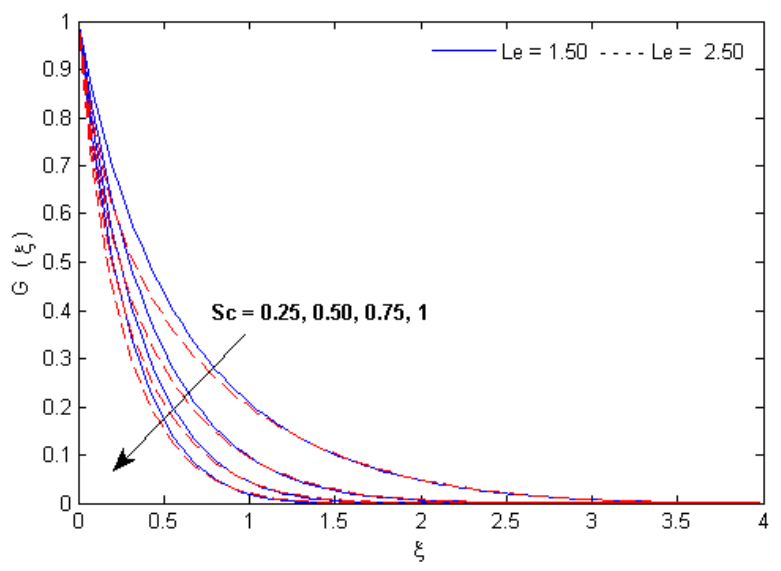

Figure 9. Motile microorganisms density profile for various $S c$ and $L e$

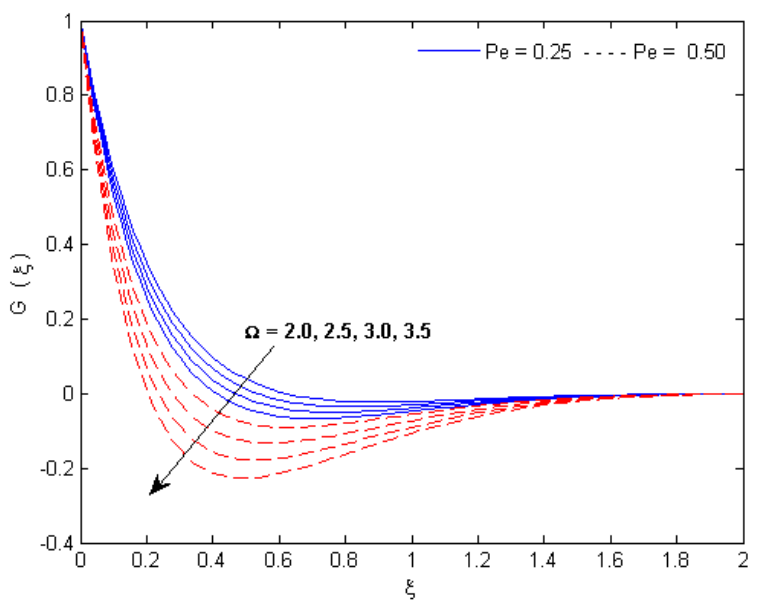

Figure 10. Motile microorganisms density profile for various $\Omega$ and $P e$

The density of motile microbes $G(\xi)$ is decreased by escalating the values of the motile microbes parameter and bioconvection Peclet number (see Figure 10). The cell swimming $W_{c}$ is directly proportional to $\mathrm{Pe}$ (Peclet number) and inversely proportional to $D_{n}$ (diffusivity of microorganisms). Peclet number is the proportion between the rates of diffusion and advection. So, an enhancement in the bioconvection $P e$ gives rise to an increment in the advective transport rate as compared to the diffusion, and consequently the flux of the self-swimming microorganisms rapidly rises. Hence growing Peclet number escalates the 
wall motile microorganisms flux, and thus depreciates the motile microbes density profile. The bioconvection Peclet number $P e$ builds the swimming rate of the motile microorganisms in regard of the fluid thus the thickness of the microorganisms is decreased close to the surface. However, one may decide that the bioconvection parameters substantially influence the motile microorganisms transport rate.

The current study of nanofluid flow with gyrotactic microbes has persuasive practical employments in numerous areas of biotechnology and science such as enzyme biosensor and microfluidics devices like micro-volumes and bacteria powered micro-mixers. The gyrotactic microbes are also helpful to improve nanofluids stability in the flow. Our results may also beneficial in improving the proficiency of microbial fuel cells. Microbial-enhanced oil recovery is also application of bioconvection phenomena where nutrients and microbes are inserted in oil bearing layer to maintain the variation in permeability.

\section{CONCLUSIONS}

A comprehensive study of bioconvection nanofluid flow comprising gyrotactic microbes through a porous media taking into account the effects of chemical reaction and heat generation is introduced. SOR technique is utilized to obtain the numerical solution against concentration, thermal energy, motile microbes density and velocity. The outcomes of the present work may be stated as:

(1). Porous medium has yields almost negligible effect on motile microbes' concentration while it significantly enhances the shear stress.

(2). The large estimations of Peclet number are linked with low diffusivity of microorganisms that causes the reduction in motile microbes' density profile.

(3). The Lewis number enhances mass transportation rate and thermophoresis parameter reduces heat transportation rate on sheet surface.

(4). The positive constant $(m)$ for nonlinear sheet, suction/injection, porosity and stretching of sheet elevate the dimensionless stream wise velocity $F^{\prime}(\xi)$.

(5). The impacts of heat generation and chemical reaction evidently disclose that there is a strong acceleration in the temperature $\theta(\xi)$ with increasing $H$-values while there is a deceleration in the concentration $\phi(\xi)$ with increasing $R_{c}$-values.

\section{ACKNOWLEDGMENT}

The authors wish to express their sincere thanks to the honorable editor and referees for their valuable comments to improve the quality of the paper.

\section{REFERENCES}

[1] Kumar, R.K., Varma, S.V.K. (2018). MHD boundary layer flow of nanofluid through a porous medium over a stretching sheet with variable wall thickness: using Cattaneo-Christov heat flux model. Journal of Theoretical and Applied Mechanics, 48(2): 72-92. https://doi.org/10.2478/jtam-2018-0011
[2] Vijaya Lakshmi, G., Anand Babu, L., Srinivasa Rao, K. (2018). MHD mixed convection stagnation point flow of nanofluid through a porous medium over stretching sheet. Int. J. Pure Appl. Maths., 118(10): 369-389.

[3] Mabood, F., Shateyi, S., Rashidi, M. M., Momoniat, E., Freidoonimehr, N. (2016). MHD stagnation point flow heat and mass transfer of nanofluids in porous medium with radiation, viscous dissipation and chemical reaction. Advanced Powder Technology, 27(2): 742749. https://doi.org/10.1016/j.apt.2016.02.033

[4] Umadevi, P., Nithyadevi, N. (2016). Magnetoconvection of water-based nanofluids inside an enclosure having uniform heat generation and various thermal boundaries. Journal of the Nigerian Mathematical Society, $\quad 35(1)$ : $\quad 82-92$. https://doi.org/10.1016/j.jnnms.2016.02.001

[5] Abbas, Z., Mushtaq, T., Shehzad, S.A., Rauf, A., Kumar, R. (2019). Slip flow of hydromagnetic micropolar nanofluid between two disks with characterization of porous medium. Journal of the Brazilian Society of Mechanical Sciences and Engineering, 41: 465. https://doi.org/10.1007/s40430019-1974-6

[6] Kuznetsov, A.V. (2010). The onset of nanofluid bioconvection in a suspension containing both nanoparticles and gyrotactic microorganisms. International Communications in Heat and Mass Transfer, 37(10): 1421-1425. https://doi.org/10.1016/j.icheatmasstransfer.2010.08.01 5

[7] HuiDS, I.A.E., Madani, T.A., Ntoumi, F., Koch, R., Dar, O. (2020). The continuing 2019-nCoV epidemic threat of novel coronaviruses to global health: the latest 2019 novel coronavirus outbreak in Wuhan, China. International Journal of Infectious Diseases, 91: 264266. https://doi.org/10.1016/j.ijid.2020.01.009

[8] Ramzan, M., Chung, J.D., Ullah, N. (2017). Radiative magnetohydrodynamic nanofluid flow due to gyrotactic microorganisms with chemical reaction and non-linear thermal radiation. International Journal of Mechanical Sciences, 130: 31-40. https://doi.org/10.1016/j.ijmecsci.2017.06.009

[9] Chakraborty, T., Das, K., Kundu, P.K. (2018). Framing the impact of external magnetic field on bioconvection of a nanofluid flow containing gyrotactic microorganisms with convective boundary conditions. Alexandria Engineering Journal, 57(1): 61-71. https://doi.org/10.1016/j.aej.2016.11.011

[10] Sk, M.T., Das, K., Kundu, P.K. (2016). Multiple slip effects on bioconvection of nanofluid flow containing gyrotactic microorganisms and nanoparticles. Journal of Molecular $\quad$ Liquids, 220: 518-526. https://doi.org/10.1016/j.molliq.2016.04.097

[11] Iqbal, Z., Mehmood, Z., Maraj, E.N. (2017). Oblique transport of gyrotactic microorganisms and bioconvection nanoparticles with convective mass flux. Physica E: Low-dimensional Systems and Nanostructures, $\quad 88$ : 265-271. https://doi.org/10.1016/j.physe.2017.01.011

[12] Atif, S.M., Hussain, S., Sagheer, M. (2019). Magnetohydrodynamic stratified bioconvective flow of micropolar nanofluid due to gyrotactic microorganisms. $\begin{array}{lll}\text { AIP } & \text { Advances, } & 925208 .\end{array}$ https://doi.org/10.1063/1.5085742 
[13] Acharya, N., Das, K., Kundu, P.K. (2016). Framing the effects of solar radiation on magneto-hydrodynamics bioconvection nanofluid flow in presence of gyrotactic microorganisms. Journal of Molecular Liquids, 222: 2837. https://doi.org/10.1016/j.molliq.2016.07.023

[14] Waqas, H., Khan, S.U., Shehzad, S.A., Imran, M. (2019). Radiative flow of Maxwell nanofluid containing gyrotactic microorganism and energy activation with convective Nield conditions. Heat Transfer-Asian Research, 48(5): 1663-1687. https://doi.org/10.1002/htj.21451

[15] Shahid, A., Zhou, Z., Bhatti, M.M., Tripathi, D. (2018). Magnetohydrodynamics nanofluid flow containing gyrotactic microorganisms propagating over a stretching surface by successive Taylor series linearization method. Microgravity Science and Technology, 30(4): 445-455. https://doi.org/10.1007/s12217-018-9600-2

[16] Ashraf, M., Bashir, S. (2012). Numerical simulation of MHD stagnation point flow and heat transfer of a micropolar fluid towards a heated shrinking sheet. International Journal for Numerical Methods in Fluids, 69(2): 384-398. https://doi.org/10.1002/fld.2564

[17] Ahmad, S., Ashraf, M., Ali, K. (2019). Numerical simulation of viscous dissipation in a micropolar fluid flow through a porous medium. Journal of Applied Mechanics and Technical Physics, 60(6): 996-1004. https://doi.org/10.1134/S0021894419060038

[18] Hildebrand, F.B. (1978). Introduction to Numerical Analysis, Tata McGraw-Hill Publishing Company, New Delhi.

[19] Zaimi, K., Ishak, A., Pop, I. (2014). Stagnation-point flow toward a stretching/shrinking sheet in a nanofluid containing both nanoparticles and gyrotactic microorganisms. Journal of Heat Transfer, 136(4): 1-9. https://doi.org/10.1115/1.4026011

[20] Ahmad, S., Ashraf, M., Ali, K. (2020). Simulation of thermal radiation in a micropolar fluid flow through a porous medium between channel walls. Journal of Thermal Analysis and Calorimetry. https://doi.org/10.1007/s10973-020-09542-w

\section{NOMENCLATURE}

$k^{*} \quad$ Darcy permeability, $N . A^{-2}$

$\tilde{\alpha}$ thermal conductivity, $W / m k$

$T_{\infty}$ ambient temperature, $K$

$C_{\infty}$ ambient concentration, $\mathrm{mol} / \mathrm{m}^{3}$
$W_{c}$ cell swimming speed, $\mathrm{m} / \mathrm{s}$

$C_{w}$ concentration at the wall, $\mathrm{mol} / \mathrm{m}^{3}$

$T_{w}$ temperature at the wall, $K$

$D_{n}$ diffusivity of microbes, $\mathrm{m}^{2} / \mathrm{s}$

$D_{B} \quad$ Brownian diffusion coefficient, $\mathrm{m}^{2} / \mathrm{s}$

$D_{T}$ thermophoresis coefficient, $M L T^{-3} \theta^{-1}$

$\tau$ nanofluid heat capacity, $j / \mathrm{kg}$

$N$ microorganisms concentration, $\mathrm{mol} / \mathrm{m}^{3}$

\section{Greek symbols}

$\tilde{\alpha}$ thermal conductivity, $W / m k$

$\rho$ fluid density, $\mathrm{kgm}^{-3}$

$v$ kinematic viscosity, $m^{2} s^{-1}$

$\mu$ dynamic viscosity, $\mathrm{Nsm}^{-2}$

$\theta$ dimensionless temperature

$\xi$ dimensionless similarity variable

\section{APPENDIX}

The values of constants $\left(A_{1}, B_{1}, C_{l}, D_{l}\right.$ etc.) in Eqns. (22)(25) are given below:

$$
\begin{gathered}
A_{1}=4+2 h^{2} P_{0}+2 h^{2} m s_{i} \\
B_{1}=2+\frac{1+m}{2} h f_{i} \\
C_{1}=2-\frac{1+m}{2} h f \\
D_{1}=2 h^{2} m+2 h^{2} P_{0} \\
A_{2}=\frac{8}{\operatorname{Pr}}-4 h^{2} H \\
B_{2}=\frac{4}{\operatorname{Pr}}+N_{b}\left(\phi_{i+1}-\phi_{i-1}\right)+(1+m) h f_{i}+N_{t} \theta_{i+1}-2 N_{t} \theta_{i-1} \\
C_{2}=\frac{4}{\operatorname{Pr}}+\frac{16}{3} N_{r}-N_{b}\left(\phi_{i+1}-\phi_{i-1}\right)-(1+m) h f_{i}+N_{t} \theta_{i-1} \\
A_{3}=4+2 h^{2} L e R_{c} \\
B_{3}=2+\frac{1+m}{2} L e h f \\
C_{3}=2-\frac{1+m}{2} L e h f \\
D_{3}=2 \frac{N_{t}}{N_{b}}\left(\theta_{i+1}+\theta_{i-1}-2 \theta_{i}\right) \\
A_{4}=4+2 P e\left(\phi_{i+1}-2 \phi_{i}+\phi_{i-1}\right) \\
B_{4}=2+\frac{1+m}{2} h S c f_{i}-\frac{P e}{2}\left(\phi_{i+1}-\phi_{i-1}\right) \\
C_{4}=2-\frac{1+m}{2} h S c f_{i}+\frac{P e}{2}\left(\phi_{i+1}-\phi_{i-1}\right) \\
D_{4}=-2 P e \Omega\left(\phi_{i+1}-2 \phi_{i}+\phi_{i-1}\right)
\end{gathered}
$$

